\title{
Pathfinder: applying graph theory to consistent tracking of daytime mixed layer height with backscatter lidar
}

\author{
Marco de Bruine $^{1,2}$, Arnoud Apituley ${ }^{1}$, David Patrick Donovan ${ }^{1}$, Hendrik Klein Baltink ${ }^{1}$, and Marijn Jorrit de Haij ${ }^{1}$ \\ ${ }^{1}$ Royal Netherlands Meteorological Institute (KNMI), Utrechtseweg 297, 3731 GA De Bilt, the Netherlands \\ ${ }^{2}$ Institute for Marine and Atmospheric Research (IMAU), Utrecht University, Utrecht, the Netherlands
}

Correspondence to: Arnoud Apituley (apituley@knmi.nl)

Received: 3 October 2016 - Discussion started: 11 November 2016

Revised: 17 March 2017 - Accepted: 27 April 2017 - Published: 30 May 2017

\begin{abstract}
The height of the atmospheric boundary layer or mixing layer is an important parameter for understanding the dynamics of the atmosphere and the dispersion of trace gases and air pollution. The height of the mixing layer (MLH) can be retrieved, among other methods, from lidar or ceilometer backscatter data. These instruments use the vertical backscatter lidar signal to infer $\mathrm{MLH}_{\mathrm{L}}$, which is feasible because the main sources of aerosols are situated at the surface and vertical gradients are expected to go from the aerosol loaded mixing layer close to the ground to the cleaner free atmosphere above. Various lidar/ceilometer algorithms are currently applied, but accounting for MLH temporal development is not always well taken care of. As a result, $\mathrm{MLH}_{\mathrm{L}}$ retrievals may jump between different atmospheric layers, rather than reliably track true MLH development over time. This hampers the usefulness of $\mathrm{MLH}_{\mathrm{L}}$ time series, e.g. for process studies, model validation/verification and climatology. Here, we introduce a new method "pathfinder", which applies graph theory to simultaneously evaluate time frames that are consistent with scales of MLH dynamics, leading to coherent tracking of MLH. Starting from a grid of gradients in the backscatter profiles, MLH development is followed using Dijkstra's shortest path algorithm (Dijkstra, 1959). Locations of strong gradients are connected under the condition that subsequent points on the path are limited to a restricted vertical range. The search is further guided by rules based on the presence of clouds and residual layers. After being applied to backscatter lidar data from Cabauw, excellent agreement is found with wind profiler retrievals for a 12-day period in $2008\left(R^{2}=0.90\right)$ and visual judgment of lidar data during a full year in $2010\left(R^{2}=0.96\right)$. These values compare favourably to other $\mathrm{MLH}_{\mathrm{L}}$ methods applied to the same
\end{abstract}

lidar data set and corroborate more consistent MLH tracking by pathfinder.

\section{Introduction}

The atmospheric boundary layer is the lowest part of the atmosphere where most of the interactions between surface and atmosphere take place. Knowledge of the processes and mechanisms in this layer is essential in meteorology and climate science. The height of the boundary layer, or mixing layer height (MLH), is an important parameter; it affects, for example, near-surface air quality, since it limits the volume of air into which pollutants are emitted, mixed and dispersed, and is therefore crucial in modelling pollution, smog and dispersion of greenhouse gases. Since the height of the mixed layer is determined by surface fluxes that drive turbulent processes (Stull, 1988), it is one of the parameters that can be used to test model representation of the energy balance against observations. MLH observations, therefore, are of key importance when testing models for a realistic representation of the atmosphere ranging from short timescales (weather forecasting) to long timescales (climate change).

Several methods exist to measure MLH. Instruments used for this include backscatter lidar (Van Pul et al., 1994; Menut et al., 1999; Eresmaa et al., 2006; Haeffelin et al., 2012; Wiegner et al., 2006), Doppler lidar (Harvey et al., 2013), radiosonde (Holzworth, 1964; Vogelezang and Holtslag, 1996; Seibert et al., 2000; Zilitinkevich and Baklanov, 2002), sodar (Beyrich, 1995) and wind profiler (Angevine et al., 1994), to name just a few. Of these instruments, the ceilometer arguably has the best combination of the number of installa- 
tions, due to the deployment in many national meteorological networks and at aerodromes (Thomas, 2016), and excellent temporal/spatial resolution - typically $24 / 7$ coverage of vertical profiles at time resolutions higher than $1 \mathrm{~min}$ and vertical resolutions of the order of $10 \mathrm{~m}$. Data sampled at this resolution in time and space, assuming adequate signal to noise, should be sufficient to track MLH, which we intend to follow at a resolution of a couple of minutes and changes in the vertical of better than $100 \mathrm{~m}$, which would be adequate for MLH studies.

Backscatter lidars and ceilometers are based on the same principles and use aerosol concentration as a tracer for MLH, which is possible because the main sources of aerosols are situated at the surface. The turbulent motions in the mixing layer cause the aerosol concentrations to be relatively well mixed within the layer, while exchange between mixing layer (ML) and free atmosphere (FA) is limited. As a result, higher aerosol concentrations are found within the mixing layer close to the ground and lower aerosol concentrations are found in the free atmosphere above. This difference in aerosol concentrations can be used to detect MLH.

Although the detection of the boundaries between atmospheric layers based on aerosol gradients has been used for many years, most of the backscatter-lidar-based techniques have difficulties to coherently track the MLH over time and may inadvertently jump between different atmospheric layers, such as residual layers. Most algorithms search for the strongest gradient in a certain time window to which the MLH is then assigned, and the next time interval is treated independently, with jumps between layers as a result. This limits the utility of these data records for deriving statistics and climatologies, as well as for processing studies and model validation/verification.

To alleviate this problem, guidance can be sought from ancillary data (e.g. Pal et al., 2013). This improves MLH retrieval at the cost of additional infrastructure and applicability will be limited to only those locations where the complete set of instruments is available. However, this also counteracts the attraction of the simplicity of the stand-alone backscatter lidar, which integrates these instruments into a network to monitor MLH over large, regional areas. Such approaches are discussed, e.g. in the COST action TOPROF (Illingworth, 2016), which aims to create an operational ground-based profiling with lidars and microwave radiometers for improving weather forecasts and E-PROFILE (EUMETNET, 2016) in which a framework is developed to exchange lidar backscatter data.

Here, we describe the new algorithm, pathfinder, which is used to track the development of the MLH during the day, based solely on single wavelength backscatter lidar data. The pathfinder algorithm is based on graph theory and the algorithm published by Dijkstra (1959) for finding the shortest path in graphs, and therefore inherently takes temporal development into account. Section 2 introduces the pathfinder method and gives a description of the different steps. Sec- tion 3 describes different instruments used for boundary layer observations as well as the methods to derive MLH that are used in this paper for validation and verification. Section 4 presents results from this new method applied to data from Cabauw. The section is concluded with a sensitivity test.

\section{Methodology}

The most common techniques used to detect MLH from lidar measurements are the gradient method (e.g. Van Pul et al., 1994; Flamant et al., 1997; Menut et al., 1999), variance analysis (e.g. Hooper and Eloranta, 1986; Menut et al., 1999) and continuous wavelet transforms (e.g. de Haij et al., 2007). Doppler lidar techniques also use aerosol backscatter, but have additional information about the velocity of particles (Harvey et al., 2013).

These techniques evaluate measurements per time step individually. Different features, like the residual layer and advected aerosol layers can cause additional strong gradients in the lidar signal besides the MLH. These additional gradients can be of same order of magnitude as the backscatter gradient on the MLH or even stronger, possibly leading to a MLH estimate alternating between these different layers. However, MLH is a relatively slowly evolving quantity and large differences between subsequent MLH estimates can be rejected. This can be accomplished by processing information from both the spatial and temporal domain simultaneously.

Alternatively, MLH can be determined manually. Even today, this remains a powerful way of determining MLH as the human brain can use knowledge on processes affecting ML development (e.g. time of sunrise and sunset or presence and type of clouds) to distinguish the correct MLH from other gradients. Plots of lidar RCS and gradient fields are visually inspected to determine exact MLH. Although this method does not give a completely independent validation for correct MLH, it does provide an opportunity to assess the performance of the pathfinder method when applied to the same lidar measurements.

\subsection{Input lidar data}

The lidar data used are the range-corrected lidar signals (RCS). This is the signal that results after subtraction of the background sky light and correction of the $z^{2}$ geometrical term in the lidar equation (e.g. Hinkley, 1976):

$$
\begin{aligned}
\operatorname{RCS}(z) & =z^{2} P(z) \\
& =z^{2} \cdot O(z) \cdot \beta(z) \cdot \exp \left(-2 \int_{0}^{z} \alpha\left(z^{\prime}\right) \mathrm{d} z^{\prime}\right),
\end{aligned}
$$

where $P(z)$ is the received power as function of distance $z$, $\beta(z)$ is the backscatter profile in $1 /(\mathrm{m} S r), \alpha(z)$ is the extinction coefficient in $1 / m$ and $O(z)$ is the overlap function between the receiving telescope field of view (FOV) and the 


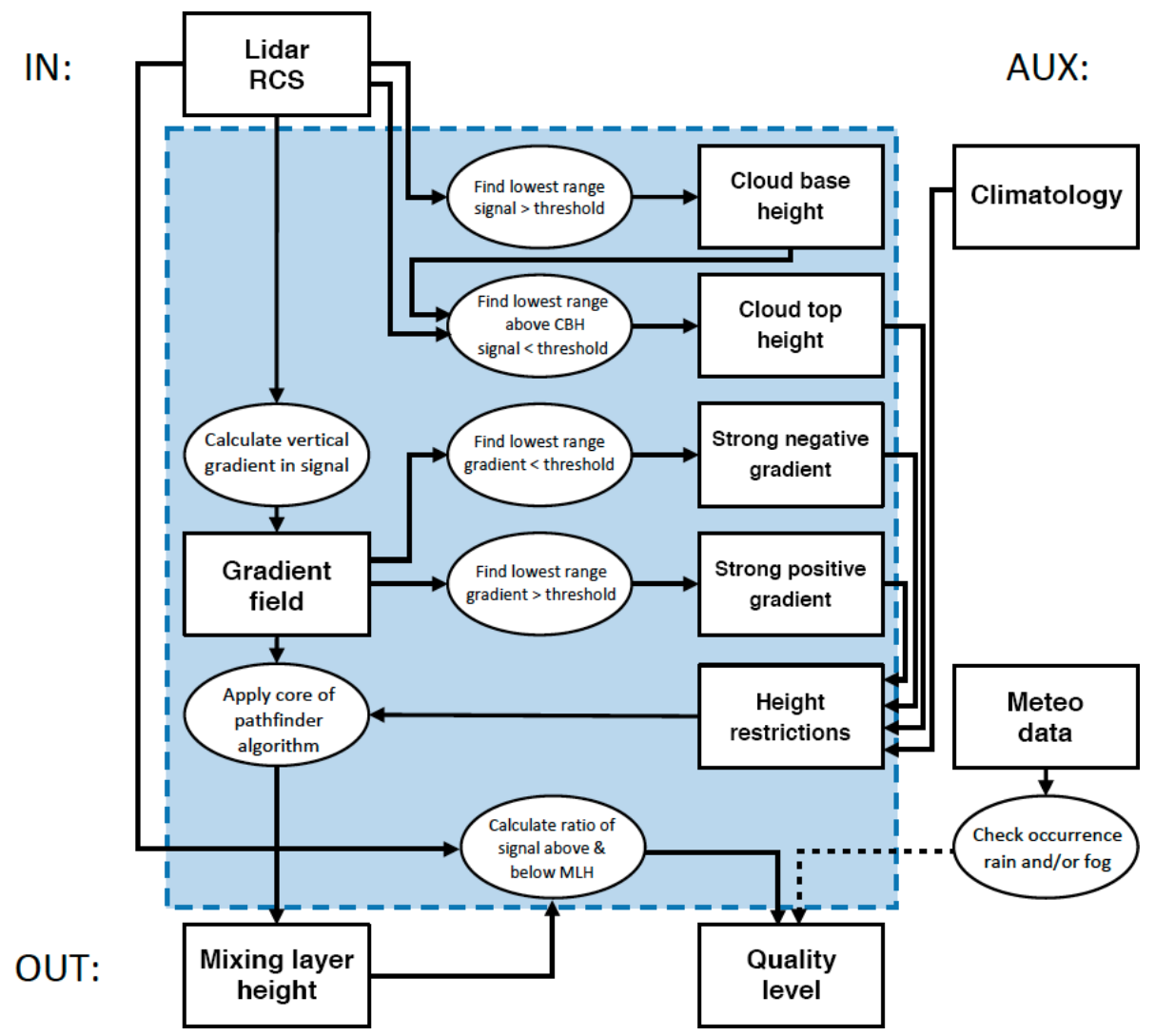

Figure 1. Flow diagram of the pathfinder algorithm. Internal operations and variables are grouped within the blue area. Input data are shown at the top and output variables at the bottom. Auxiliary data are displayed on the right. The use of collocated meteorological data is optional

area illuminated by the outgoing laser beam. For the MLH retrieval, we will only be looking at the uncalibrated properties of the lidar signal, and in particular, the vertical gradient in RCS, indicated as RCS'. Calibration constants are therefore cancelled out. The overlap function, however, is important, as it increases from zero to unity from the ground up to the altitude of full overlap: $z_{\mathrm{f}}$. Above $z_{\mathrm{f}}$ we assume $O\left(z>z_{\mathrm{f}}\right)=1$. In the region of incomplete overlap below $z_{\mathrm{f}}, \mathrm{RCS}^{\prime}$ will be influenced by $O$ by adding a systematic positive contribution to the gradient. A further consequence of the lidar instrument geometry is that no lidar signal is obtained at very close ranges below $z_{b}$. In the lowest range between the instrument and $z_{b}$, the lidar is essentially blind.

\subsection{The pathfinder method}

The pathfinder method starts with a time series of RCS data as defined in Eq. 1, which is also represented in Fig. 3. Since the data can include atmospheric circumstances under which MLH detection may not be feasible (i.e. fog, intense precipitation, low clouds), some pre-selection of data is needed, such as cloud screening. So first, parts of the data in time and/or height will be excluded for analysis based on four different guiding restrictions as explained in Sect. 2.2.1. In the second stage, as the core of the algorithm, the vertical gradients in the lidar data are computed and the gradient data $\mathrm{RCS}^{\prime}$ are transformed into a mathematical graph in which all measurement points are represented by a node (vertex) and the connections (edges) are constructed based on costs calculated from the measurements. Dijkstra's (1959) algorithm is then applied to find the combination of measurement points representing the temporal evolution of the MLH. In the last stage a quality label is assigned to the MLH estimates based on the ratio of RCS above and below the MLH estimates. A flow diagram of the algorithm is given in Fig. 1.

\subsubsection{Guiding restrictions}

Although the ML can exhibit many different features, the occurrence of clouds, residual layers and advected air masses are always situated above or coincident with of the ML. In the lidar measurements these can be detected by high RCS (clouds) and gradients in the RCS (residual and advected layers). Pathfinder uses this to construct altitude restrictions before estimating MLH. These restrictions are based on cloud top height, negative and positive gradients. An additional restriction is the local MLH climatology. 
(a)

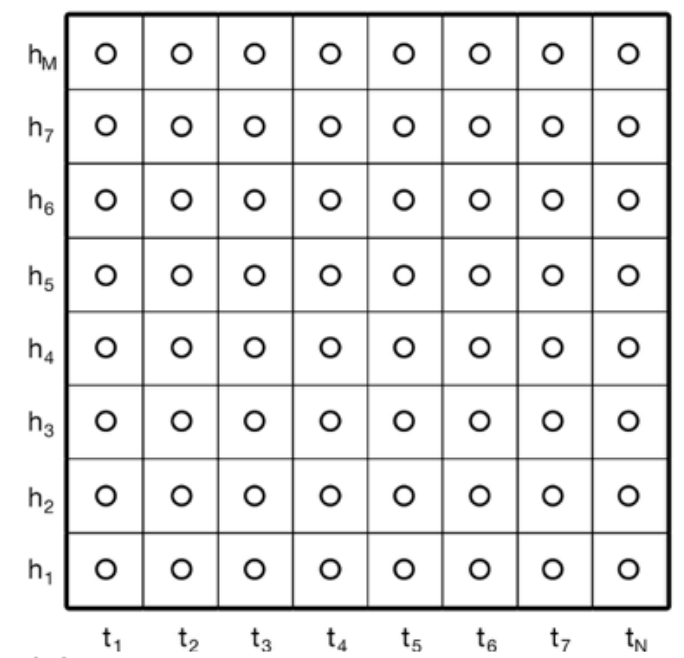

(c)

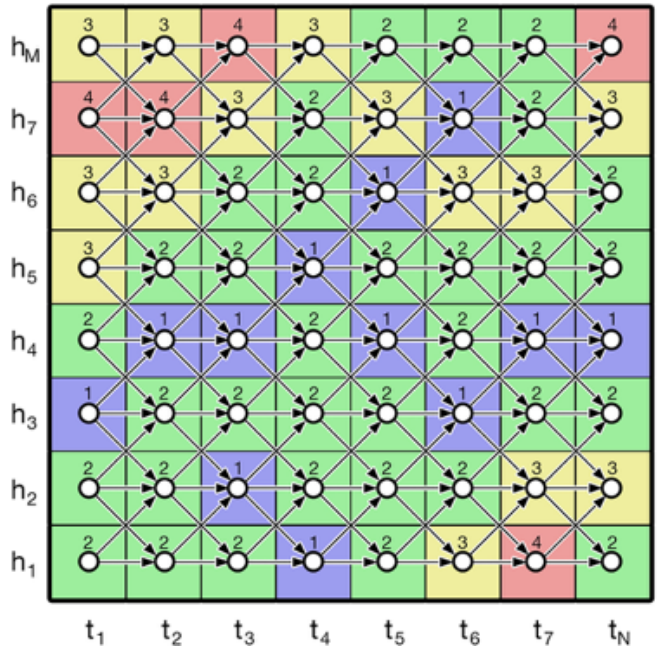

(b)

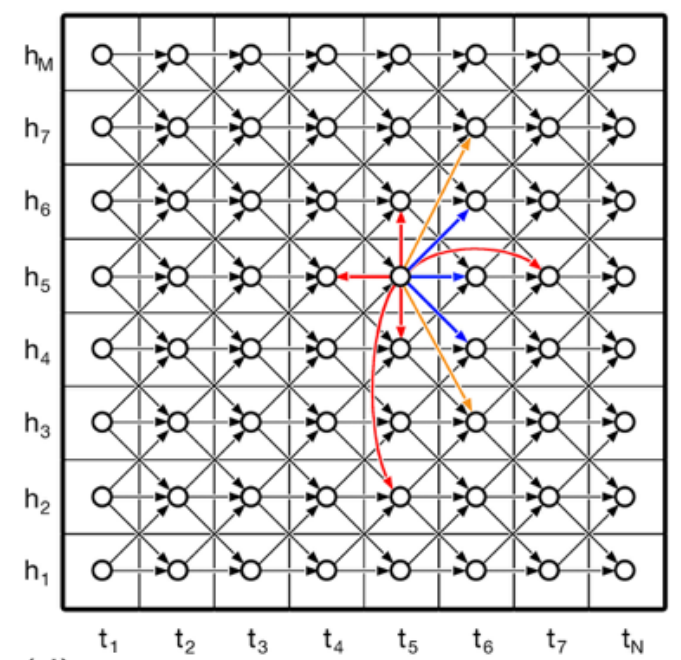

(d)

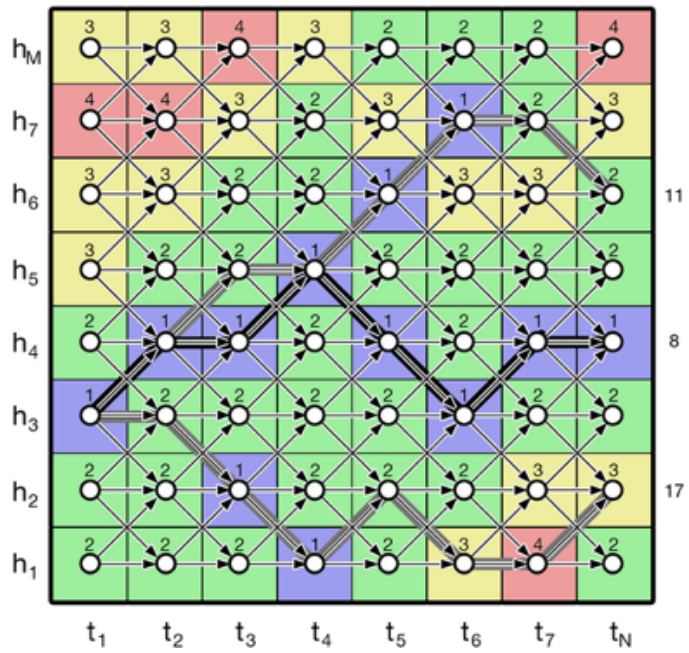

Figure 2. Overview of the translation of data into a mathematical graph and subsequent steps to determine MLH. (a) One-to-one translation of data points to graph vertices. (b) Grid of connections between vertices, for a single vertex allowed connections are shown in blue, examples of forbidden connections shown in orange and red. (c) Assignment of costs to the graph connections based on RCS vertical gradients. (d) Selection of correct path with Dijkstra's algorithm, with three possible paths originating from the same point. Their total cost is displayed at the right. The middle path will be selected as the MLH development estimate as this is the path with the lowest possible cost.

The pathfinder algorithm restricts the maximum height of the MLH to the top of the first cloud layer. Cloud detection in pathfinder is simple yet effectively based on the magnitude of RCS, which is possible due to the high backscatter on cloud droplets compared to aerosols. For each time step, the lowest altitude at which RCS exceeds a prescribed threshold is marked as cloud base height (CBH). After that, the lowest altitude above the $\mathrm{CBH}$ at which RCS drops below the same threshold is marked as cloud top height (CTH). Note that CTH is an apparent cloud top height. For optically thick clouds, the lidar signal might not penetrate the complete cloud and the signal might drop below the threshold value before reaching the actual cloud top. Therefore, it is not certain that CTH marks the correct cloud top. Nevertheless, we do not limit the search range to the cloud base, because, in case of shallow cumulus clouds, the air in the cloud is part of the boundary layer. The apparent cloud top marked as CTH is always closer to the correct cloud top and MLH than the cloud base.

Even though RCS ${ }^{\prime}$ at the top of the ML can be weak or even absent at times, a strong gradient is always an indication of a separation of air masses. In case of multiple strong gradients, the search range can be restricted to the altitude of the gradient closest to the surface. Based on this, pathfinder limits the search range to the lowest altitude where RCS' exceeds a prescribed negative or positive value. These thresholds do not necessarily have the same magnitude. Because the boundary of the ML is not so well established in the 


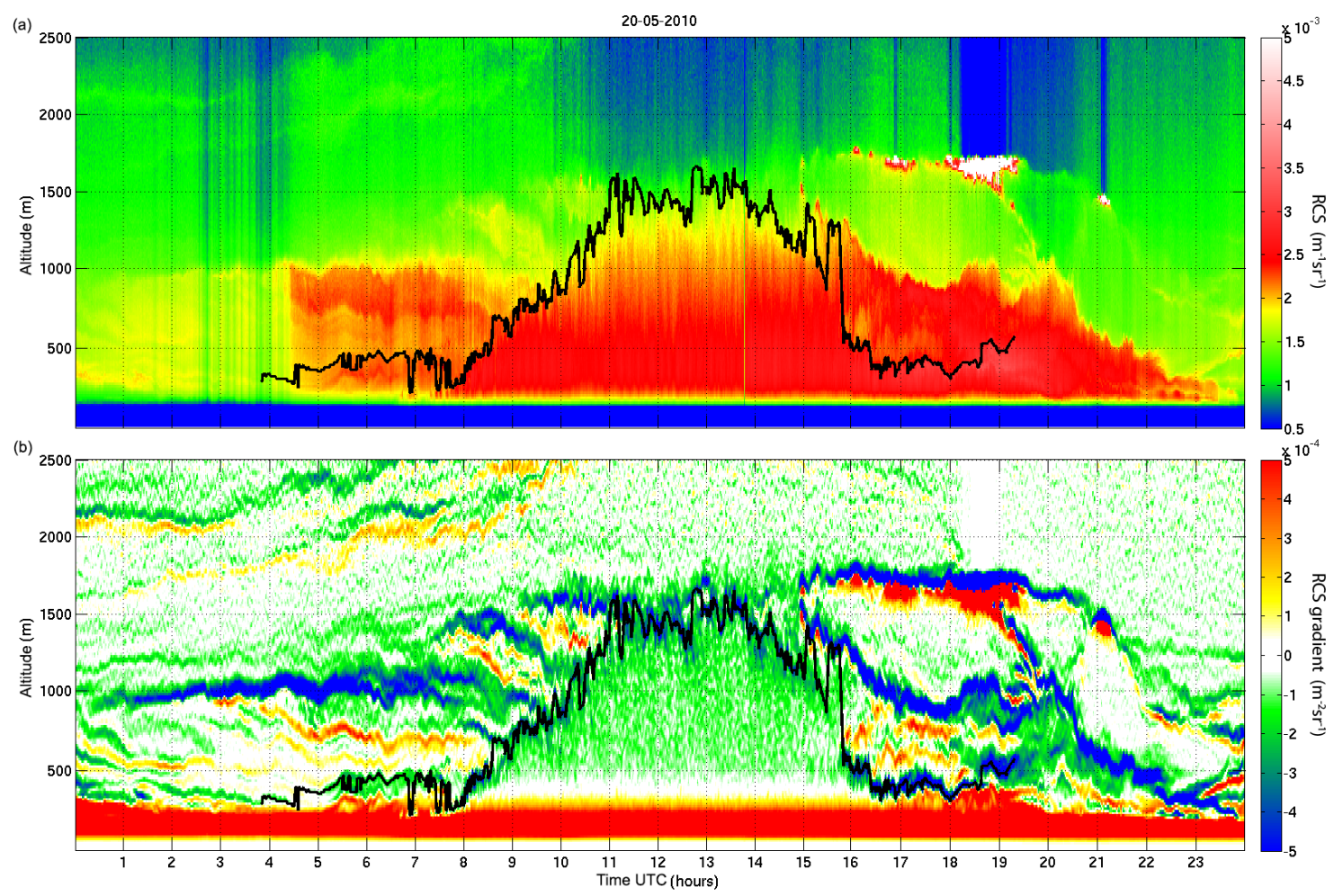

Figure 3. (a) A full diurnal cycle of backscatter lidar profiles from the ALS450 system at Cabauw on 20 May 2010. The data presented are the range-corrected signal, RCS. The black line indicates pathfinder MLH solution. (b) The gradient field calculated from the backscatter lidar data.

morning, a stricter threshold can be used before the convective period. For an overview of thresholds used for the ALS450 UV-Lidar at Cabauw, see Table 2. To prevent exclusion of ML clouds, interaction between the positive gradient and previously described cloud detection is needed. When a cloud base is found within $300 \mathrm{~m}$ of a positive gradient, the search range is extended to the cloud top. This range is based on the typical scale of increased RH near clouds.

From literature, the scales associated with the ML in the midlatitudes are well known. For example, the ML in the Netherlands rarely extends above about $750 \mathrm{~m}$ during nighttime and about $3000 \mathrm{~m}$ during daytime. This is implemented in the algorithm by restricting the search range during the morning period to that of the night period. On the onset of the convective period, a linear increase of $2.5 \mathrm{~ms}^{-1}$ is allowed, until the maximum altitude for daytime is reached. For the remainder of the day, the search range is limited by the daytime maximum.

Restriction to the exact altitude of cloud top or gradient might cause the exclusion of the actual MLH from the search range if the feature triggering a restriction is coincident with MLH. To prevent this from happening, the restrictions are relaxed in altitude by $75 \mathrm{~m}$. Additionally, to prevent a single noisy measurement or inhomogeneity to disturb the algorithm, the restrictions are also relaxed in time by $2 \mathrm{~min}$.
Table 1. Main characteristics of Leosphere ALS450.

\begin{tabular}{lll}
\hline Emission & Wavelength & $355 \mathrm{~nm}$ \\
& Power & $16 \mathrm{~mJ}$ pulse $^{-1}$ \\
& PRF & $20 \mathrm{~Hz}$ \\
& Divergence & $<0.5 \mathrm{mRad}$ \\
\hline Detection & Wavelength & $355 \mathrm{~nm}$ \\
\hline
\end{tabular}

Settings of the algorithm related to these points are included in Table 2. Note that our own experience with the application of the algorithm was limited to a single instrument, so we cannot exclude the possibility that additional parameters may have to be introduced to apply pathfinder to other instruments. The numerical thresholds for clouds and positive and negative gradients are instrument specific because the backscatter intensity depends on the type of laser used and accompanying specifications. The strength of smoothing applied to the data is related to the signal-to-noise ratio of the lidar. The minimum altitude to search for MLH is determined by the lidar overlap function and is also instrument (and possibly even serial number) specific. The convective delay depends on the geographic location of the measurements. Likewise, the maximum altitudes during night and day are deter- 
Table 2. Overview of pathfinder parameters applied to Leosphere ALS450 lidar data.

\begin{tabular}{ll}
\hline Parameter & Value \\
\hline $\begin{array}{ll}\text { Gaussian smoothing } \\
\text { Convective delay }\end{array}$ & $\begin{array}{l}\sigma=1.1 \text { range gates } \\
3 \mathrm{~h}\end{array}$ \\
\hline Guiding restrictions & \\
\hline Clouds & $>5 \times 10^{-3} \mathrm{~m}^{-1} \mathrm{Sr}^{-1}$ \\
Negative gradient & $>5 \times 10^{-4} \mathrm{~m}^{-2} \mathrm{Sr}^{-1}$ \\
Positive gradient morning & $<-1 \times 10^{-5} \mathrm{~m}^{-2} \mathrm{Sr}^{-1}$ \\
Positive gradient day & $<-2 \times 10^{-4} \mathrm{~m}^{-2} \mathrm{Sr}^{-1}$ \\
Maximum altitude night & $750 \mathrm{~m}$ \\
Maximum altitude day & $3000 \mathrm{~m}$ \\
Minimum altitude & $175 \mathrm{~m}$ \\
\hline Graph & \\
\hline Window size & $15 \mathrm{~min}$ \\
Vertical range 1-to-1 & $75 \mathrm{~m}$ \\
Vertical range total & $900 \mathrm{~m}$ \\
\hline
\end{tabular}

mined by the environment and referred to as "climatology" in the text.

\subsubsection{Applying graphs}

Tracking the evolution of MLH is essentially selecting a series of points with corresponding time and altitude from a data set, based on certain criteria - much like planning an optimal route on a map. The mathematical representation of information in graphs is an excellent tool for this purpose. To apply graphs for MLH tracking from lidar measurements, we define the following four steps. First, a graph is set up with each point in the data set as vertices. Secondly, connections between these vertices are made so that any collection of connected vertices (hereafter called "path") in the graph represents a physically possible MLH evolution. Third, costs are assigned to the connections. Finally, the graph is searched by Dijkstra's shortest path algorithm to select the optimal path following the MLH.

In the first step, a graph is created following the structure of the corresponding data set. Every point in the data set is translated into a vertex, regardless of the actual values in the data set. For a data set with $N$ time steps and $M$ range gates, this will lead to a graph with $N \times M$ vertices, illustrated in Fig. 2a. In this stage, the graph is unstructured and the vertices unconnected.

To restrict possible paths in the graph to a physically sensible MLH evolution, connections between vertices represent specific conditions. The first condition is that vertices only connect to vertices of the next time step. Connections to vertices of the same time step, previous time steps or more than one time step away are not allowed. These excluded connections are represented by the red arrows in Fig. $2 b$.
Next, only connections to vertices within a restricted vertical range are allowed - represented by the additional orange and blue arrows in Fig. 2b. The blue connections are allowed, but the orange connections are not because they exceed the vertical range limit. Note that the representation in Fig. 2 is simplified and the actual vertical restriction can include multiple range gates depending on the combination of vertical resolution of the data and chosen vertical restriction. In our implementation, the maximum allowed MLH growth rate is $2.5 \mathrm{~ms}^{-1}$, so the allowed range is $75 \mathrm{~m}$ when the time between measurements is $30 \mathrm{~s}$. An additional restriction is posed on a timescale of $15 \mathrm{~min}$. Over this time window, connections to vertices exceeding a growth rate of $1 \mathrm{~ms}^{-1}$ are not allowed. This growth rate is larger than values found, e.g. by Baars et al. (2008), who reports values of $1 \mathrm{~km} \mathrm{~h}^{-1}$ or $0.278 \mathrm{~ms}^{-1}$ only in extreme cases. However, these values represent mean growth rates and do not represent timescales resolving individual rising thermals. Implementing lower values caused problems in the pathfinder algorithm when tracking fast MLH development in the morning.

In the third step, costs are assigned to the connections in the graph to determine which path represents the MLH from the collection created in the previous steps. For pathfinder, costs $C$ are based on the vertical gradient $\mathrm{RCS}^{\prime}$ :

$C=-\left(\frac{\partial}{\partial z} \mathrm{RCS}\right)^{-1}$

Connections pointing to a certain vertex are assigned a cost corresponding to the point in $\mathrm{RCS}^{\prime}$ represented by that vertex. Using this conversion, strong negative $\mathrm{RCS}^{\prime}$ corresponds to low costs and vice versa. Consequently, the path with the lowest total cost will include the vertices with strongest negative gradients. The assignment of costs is shown in Fig. 2c, where the colours blue, green, yellow and red represent $\mathrm{RCS}^{\prime}$. Blue is the strongest negative gradient, green and yellow are intermediate and red represents the weakest negative gradient. Note that the minus sign in the conversion Eq. (2) is needed for positive costs for negative gradients, since $\mathrm{Di}$ jkstra's algorithm needs positive costs. Whenever negative costs still occur, these are given a prescribed fill value.

In the final step, Dijkstra's shortest path algorithm is applied to select the optimal MLH path. This method efficiently determines the path with the lowest total cost originating from a specific vertex in the first time step to one of the vertices in the last time step satisfying the above-mentioned conditions (Fig. 2d).

Theoretically, any data set of arbitrary length can be translated into a graph and analysed simultaneously. However, even with the relative efficiency of Dijkstra's algorithm, this is unpractical and would lead to long processing times. Therefore, it was decided to split data into multiple time windows and apply the method to these windows separately. This improves the processing times substantially, making the 
method available for near-real-time MLH tracking. Whereas computational cost determines the upper limit of the window size, the lowest limit is determined by the typical timescale within the ML. For the results shown in Sect. 4, measurements are combined in time windows with a size of $15 \mathrm{~min}$, which is large enough to capture several rising thermals simultaneously. For a continuous series of MLH estimates, the last time step in a time window overlaps with the first time step of the subsequent time window. The MLH estimate in this time step is used as the starting point of the next time window. Without a preceding time window, the first window on a day has no normal starting point. Instead, for the first time window the vertex in the first time step with the strongest negative gradient is used as the starting point.

\subsubsection{Quality flagging}

In the final stage of the pathfinder algorithm, a quality flag is added to each point of the MLH estimates. The quality criterion is based on the ratio $R$ of the RCS above and below the estimated MLH, similar to the quality flag used in de Haij et al. (2007) and Morille et al. (2007). The ratio is calculated as follows:

$r_{Q}=\frac{\overline{\operatorname{RCS}}(z=\mathrm{MLH}, \mathrm{MLH}+150 \mathrm{~m})}{\overline{\operatorname{RCS}}(z=\mathrm{MLH}-150 \mathrm{~m}, \mathrm{MLH})}$,

where $\overline{\mathrm{RCS}}$ indicates the average RCS over the indicated range interval.

Since we expect RCS to be smaller above MLH than below it, $r_{Q}$ is expected to be less than 1 . If the algorithm marked an erroneous, noise induced gradient within the ML or FA, the backscatter above and below the estimate are comparable and the ratio is close to 1 . However, if the top of the residual layer is marked as MLH or aerosol concentrations in the ML as low, this ratio can also be small. Consequently, a high ratio is an indication of an incorrect MLH estimate, but a small ratio is no guarantee the MLH is correct. As a threshold, ratios above 0.9 are considered an indication of an incorrect MLH estimate.

\section{Instrumentation}

\subsection{Cabauw site}

The instruments for this study were located at the Cabauw Experimental Site for Atmospheric Research (CESAR; Apituley et al., 2008) in the Netherlands, where a suite of operational and research mode remote sensing and in-situ measurements are performed to comprehensively characterise the atmospheric column. At CESAR, multiple instruments are operated that can be used to validate and verify the MLH retrievals described here.

For the development of pathfinder, a continuous data set was needed from a backscatter lidar with sufficient signal to noise. This was provided by the ALS450 described in Sect. 3.2. For comparison with other, independent, MLH methods (Sect. 3.3 and 3.4), periods were sought for, where multiple instruments were operating simultaneously. The instruments and methods used are briefly introduced below.

\subsection{Backscatter lidar}

The main instrument used for the development and testing in this study was a single wavelength backscatter lidar, the Leosphere ALS450, operating at $355 \mathrm{~nm}$. This particular instrument also measures depolarisation (Donovan and Apituley, 2013), but this information is not used here. The instrument is running 24/7 and records an averaged lidar signal every $30 \mathrm{~s}$ at a spatial resolution of $15 \mathrm{~m}$. The ALS450 has a maximum operational vertical range for clouds and aerosols of $15 \mathrm{~km}$ during night-time and a reduced range of $8 \mathrm{~km}$ during the day due to remaining solar background. Main characteristics are tabulated in Table 1 . The region of incomplete overlap between the probing laser beam and the receiving telescope extends up to about $500 \mathrm{~m}$. All data from the ALS450 are available from the CESAR database (Baltink, 2016).

The atmospheric clear air attenuation is not entirely negligible at $355 \mathrm{~nm}$, and adds a negative contribution to the gradient. However, we will neglect contribution to the gradient, as it is very smooth compared to the gradients from the aerosol backscatter.

The ALS450 has been operational at Cabauw since 2007. Unfortunately, significant gaps exist in the instruments' data record due to frequent instrument failure. The continuity of data coverage was best in 2010 , which is why this data period was selected, providing a full annual cycle to be studied.

Also installed at Cabauw is an Impulsphysik LD40 ceilometer, which is part of the Dutch ceilometer network, from which MLH is routinely derived (de Haij et al., 2007). The LD40 operates at $905 \mathrm{~nm}$ and has a maximum operational vertical range for clouds of about $10 \mathrm{~km}$. However, for aerosols the range is often limited to $2 \mathrm{~km}$ or less due to the low signal-to-noise ratio (SNR). Pathfinder was applied to LD40 data, but due to the limited SNR no useful intercomparison could be made to the ALS450 or other instruments. Currently, the LD40 is being decommissioned and replaced by the more capable Lufft CHM15k for the entire network in the Netherlands. However, the data could not be included in this study, but is the subject of future work.

\subsection{Wind profiler}

The wind profiler/RASS (Radio Acoustic Sounding System) is a clear-air radar measuring Doppler shift to collect information on the vertical wind profile. The profiler at Cabauw operates at $1290 \mathrm{MHz}$. To measure the wind profile it executes a measuring cycle consisting of measurements in four different oblique directions ( $15.5^{\circ}$ off-zenith) and one in the vertical. Combining the measurements from the different di- 
rections gives horizontal and vertical wind speeds. MLH detection from the wind profiler is possible since the intensity of the returning signal primarily depends on inhomogeneities in the atmospheric moisture and temperature caused by turbulence. The SNR is directly proportional to these inhomogeneities, captured in the refractive index structure function $C_{n}^{2}$ (Ottersten, 1969). Due to the entrainment at the MLH, $C_{n}^{2}$ exhibits a (local) maximum at this height. The width of the Doppler spectrum is also used in the MLH detection. The spectral width is related to the differences in wind speed in the probed volumes, is smaller in the entrainment layer than in the mixing layer itself and increases again into the free atmosphere. Therefore, MLH is associated with a minimum in the spectral width. Results for the $\mathrm{MLH}_{W}$ used in this paper use the method described by Angevine et al. (1994). A data set processed for MLH retrieval overlapping with ALS450 data was available for the IMPACT campaign in 2008 (Roelofs et al., 2010).

\subsection{Radiosonde}

Radiosonde data from Vaisala RS92-GDP measuring profiles of relative humidity, temperature, pressure and wind are used from the IMPACT campaign (Roelofs et al., 2010) that were launched from the Cabauw site. These sondes were used in the comparison between lidar, wind profiler and sonde (Sect. 4.3). Note that the sondes we used here for best collocation are not the routine radio sonde data from De Bilt, at about $20 \mathrm{~km}$ from Cabauw. However, for the full-year analysis presented in Sect. 4.4 we considered the De Bilt sondes.

Several methods exist to calculate MLH from the radiosonde measurements $\left(\mathrm{MLH}_{S}\right)$, including the parcel method (Holzworth, 1964), lapse rate method (Hayden et al., 1997) and Richardson bulk method (Vogelezang and Holtslag, 1996; Seibert et al., 2000). Here, we will use the Richardson bulk method since it takes into account both instability and wind shear.

\section{Results}

The performance of pathfinder is demonstrated in a couple of case studies: under clear-sky conditions in Sect. 4.1 and in more complex atmospheres with clouds and precipitation in Sect. 4.2. Subsequently, our new method is intercompared with MLH estimates from other instruments in Sect. 4.3. To test the applicability of pathfinder semi-operationally and to include a wide range of weather conditions, a comparison with manual MLH estimates for a full year is discussed.

Furthermore, the same data set is also processed by the STRAT2D method (Menut et al., 1999; Morille et al., 2007; Haeffelin et al., 2012) in Sect. 4.3.2. This method was selected, since the STRAT2D code has been made available to the community at large, specifically to be adapted and applied to lidar instruments at the disposal of interested inves- tigators. Therefore, pathfinder and STRAT2D applied to the same data provide an insight in algorithm differences, without introducing doubts about differences between various instrument characteristics.

The following data are all based on measurements of the Leosphere ALS450 at Cabauw, for which the pathfinder tuning parameter values are listed in Table 2.

\subsection{Clear sky}

The first case study is the evolution of $\mathrm{MLH}_{\mathrm{L}}$ on 20 May 2010: a clear-sky day with a well-developed afternoon ML. The measured backscatter and corresponding vertical gradients are shown in Fig. 3. Also shown is the pathfinder MLH estimate from sunrise to sunset. To point out different features of the algorithm, four regions will be highlighted: (1) the period between sunrise and the onset of convection, (2) the morning growth of the ML, (3) the plateau during the middle of the day and (4) the breakdown of the ML and transition into the night-time boundary layer. These regions can be seen in Fig. 4.

From sunset it takes several hours before the surface temperature is high enough to produce convection visible in lidar observations. With the complete ML below the lidar detection range $z_{b}$, the solution tracks a gradient in stratification of the residual layer. Around 07:00 UTC, the ML rises above $z_{b}$ and becomes visible to the lidar. The solution indicates the correct altitude as MLH. As the layer moves up and down, it again sinks below the detectable range, but when the layer reappears after some $30 \mathrm{~min}$, the solution again indicates the correct height for MLH.

The greatest challenge in deriving MLH from lidar measurements is to separate the gradient associated with the ML from the gradients in the residual layer. The pathfinder method will ignore additional layers when these are relatively far from the MLH. However, when an additional strong gradient exists close to the MLH, it might be included in the MLH estimate. The algorithms' decision depends on the proximity of the gradients and the ratio of their relative strength. For the solution to shift to a different layer it has to transition several points of weak gradients and receives a penalty for this in the form of a higher path cost. A relatively strong gradient on a residual layer can outweigh this penalty and still cause a shift in the path with the lowest total path cost. Figure $4 \mathrm{~b}$ shows that for the major part of the morning transition the correct MLH is indicated. Only around 08:45 UTC is the solution indeed triggered on a gradient in the residual layer, when the gradient on the real MLH becomes weaker.

Although individual rising thermals are also visible during morning growth, the differences between them are even more pronounced at midday when the height of the ML is more or less stable. As shown in Fig. 4c, the difference in altitude between subsequent thermals is of the order of $100-500 \mathrm{~m}$, while the average MLH is nearly constant. The vertical lim- 

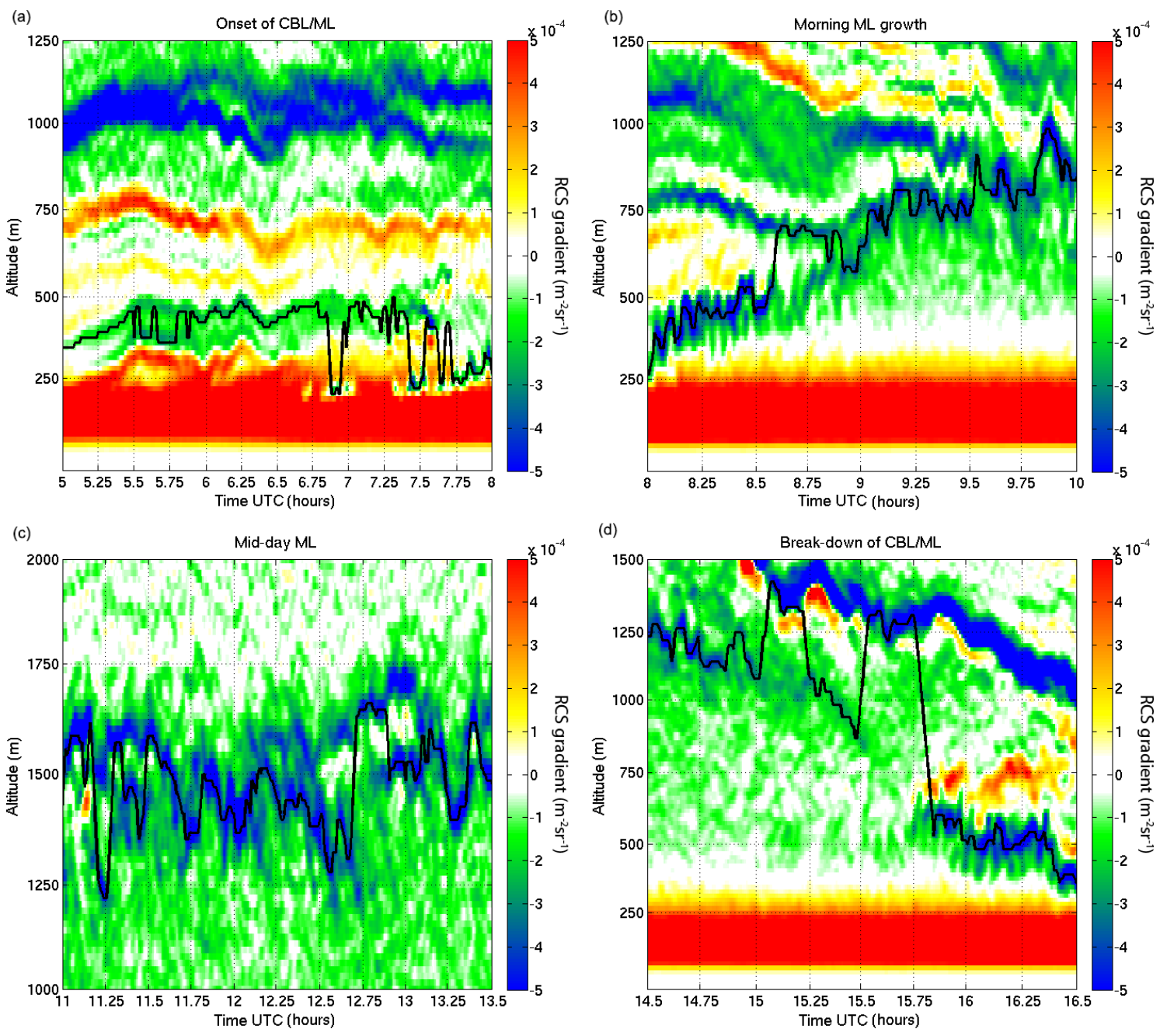

Figure 4. Highlights of the MLH evolution at Cabauw on 20 May 2010. See text in Sect. 4.1. Gradients in RCS and pathfinder MLH estimates during a clear-sky day. These include (a) the period between sunrise and onset of the ML, (b) the morning growth of the ML, (c) the midday structure of the MLH and (d) the break-down of the ML. Time is indicated in decimal hours.

itation of $75 \mathrm{~m}$ between time steps is enough to follow the differences between thermals, such as the sudden decrease around $11: 15$ and 12:45 UTC.

\subsection{Complex atmospheres}

Even though a clear-sky day gives a good insight into different ML features, completely cloud-free days are scarce in the Netherlands. The presence of clouds influences the evolution of the ML (e.g. by blocking incoming solar radiation) and causes additional gradients which can distract the algorithm from the correct solution. As an example for this, the next case study treats a day with abundant fair-weather cumulus clouds. As can be seen in Fig. 5, two layers of clouds are present, mainly stratocumulus above cumulus clouds forming on top of the ML.

Around sunrise and sunset, the two cloud layers are well separated. Pathfinder correctly designates MLH to the top of the lowest cloud layer. This is mainly due to the guiding restriction that excludes measurements above the first cloud layer. However, this is a broken cloud deck and the guiding restrictions cannot exclude the stratocumulus clouds for all time steps. It is the combination of the guiding restriction together with the limited vertical search range that ensures correct tracking of the MLH even for broken cloud layers.

With the growth of the ML, the distance between the two cloud layers decreases up to a point where the two can no longer be distinguished when the signal extinction is too strong in the cumulus layer. During these periods, the solution tracks the top of the stratocumulus as MLH, leading to short peaks in MLH, e.g. around 10:00 UTC.

The limited vertical searching reach causes MLH to be assigned to a cloud layer, which is beneficial for tracking broken cloud decks. However, an example of the solution tracking the wrong cloud layer can be seen in Fig. 6 between 15:45 


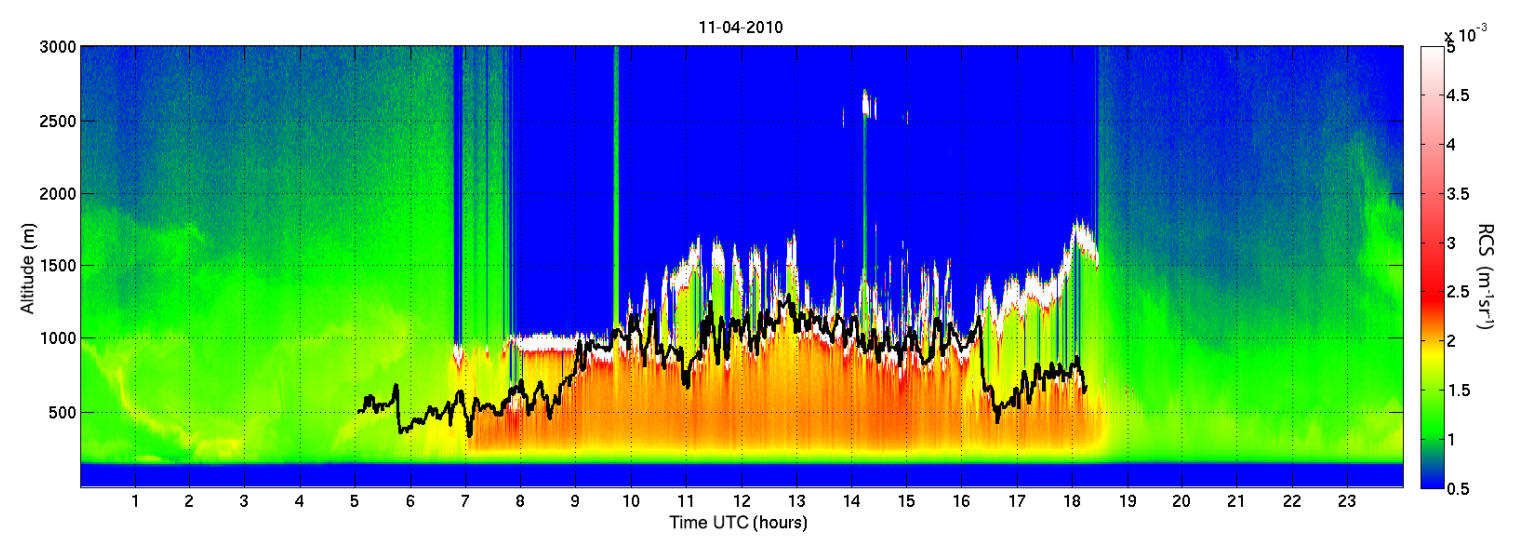

Figure 5. Lidar RCS together with pathfinder MLH estimate on 11 April 2010. A day with cumulus cloud forming on top of the ML with an additional stratocumulus cloud layer above.
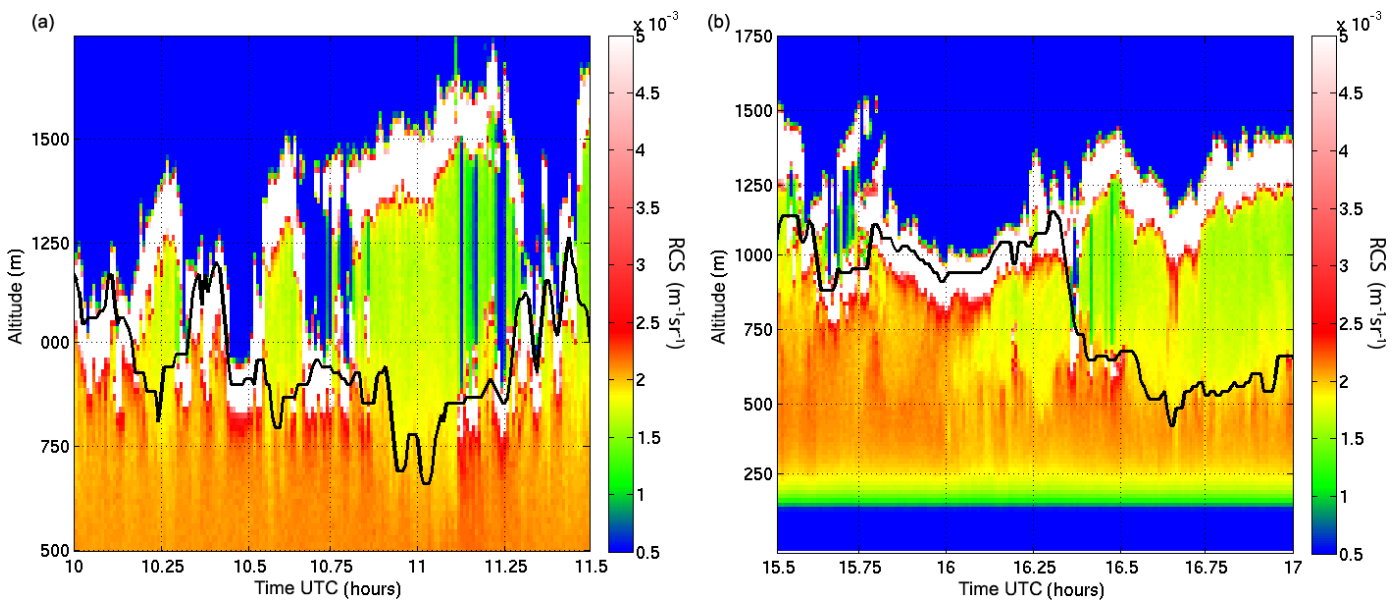

Figure 6. Highlights of the MLH estimate found by the pathfinder algorithm. See text in Sect. 4.2. Shown are (a) difficulties in distinguishing ML and non-ML clouds and (b) commitment to a cloud layer that detaches from the ML.

and 16:45 UTC. The ML decreases in depth, but the cloud layer remains at the same altitude. The pathfinder algorithm keeps tracking the strong gradients on the apparent cloud tops instead of the MLH. Because pathfinder is designed to track gradients close to adjacent gradients, the algorithm will not divert from a cloud layer until either strong gradients or clouds trigger the guiding rules.

The last two cases consider the ML during precipitation events. An example of a day with (heavy) precipitation is 8 June 2010, seen in Fig. 7. During and after precipitation, the lidar observations are affected by water on top of the instrument. Only the strong backscatter on raindrops is strong enough to be distinguished from the background and noise. Therefore, a lidar-based MLH estimate in these periods is unreliable if not impossible. Between 06:30 and 08:30 UTC the estimates fluctuate between 150 and $750 \mathrm{~m}$, the complete range allowed by the guiding restrictions. During rain between 14:30 and 15:30 UTC, the signal is too low to calculate reliable MLH estimates.
Another day with frequent precipitation is 20 June 2010, seen in Fig. 8. A characteristic of this day was that most of the precipitation evaporated before reaching the ground. Only $0.2 \mathrm{~mm}$ of rain was recorded at Cabauw that day. Without the observations affected by water on top the instrument, this day gives an insight into the effects of precipitation on the ML and the MLH solution of pathfinder under these conditions. Evaporating precipitation can be grouped into two categories: evaporation in the lidar overlap region or well above it.

The period between 14:00 and 15:00 UTC is an example of precipitation reaching the overlap region. Because the raindrops evaporate on their way down, the highest droplet concentration and accompanying backscatter are strongest near the cloud base. Consequently, the RCS increases with altitude and no negative gradient is found until the apparent cloud top is reached. This altitude is then indicated as MLH.

Precipitation evaporating well above the overlap region can be seen in Fig. 9b. Around 11:15 UTC, precipitation ap- 


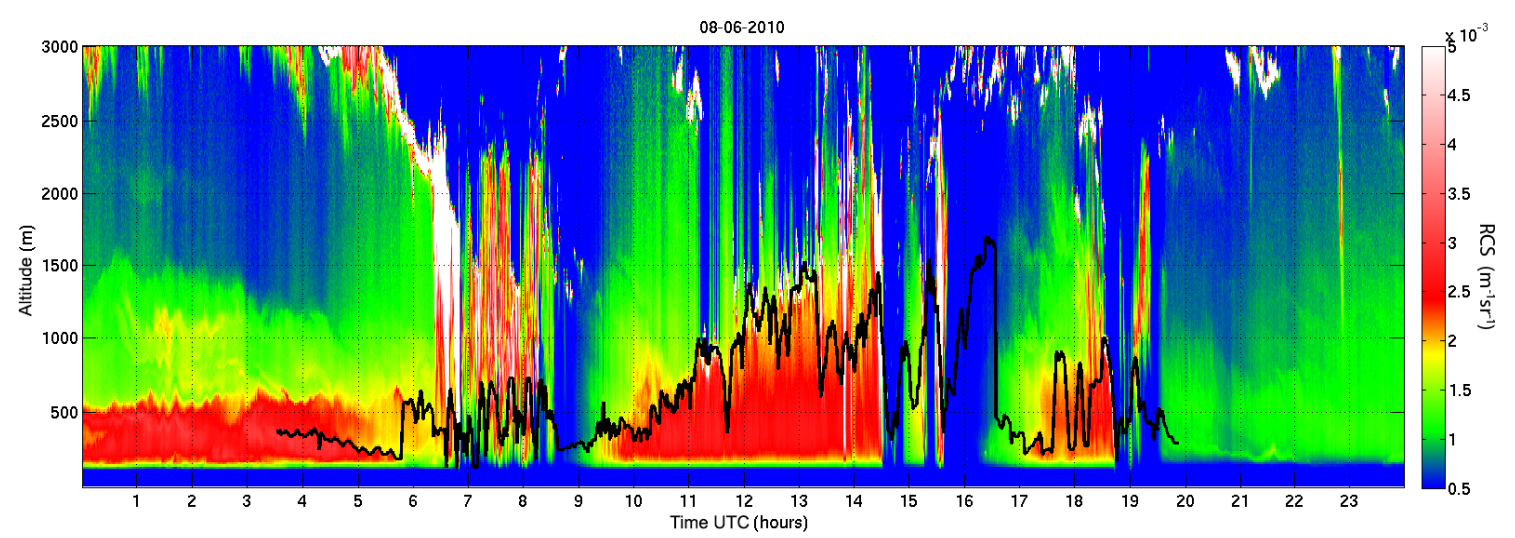

Figure 7. Lidar RCS together with pathfinder MLH estimate on 8 June 2010. A day with varying cloud types and changing precipitation rates, including some showers between 06:00 and 09:00, 15:00 and 16:00 and at 19:00 UTC.

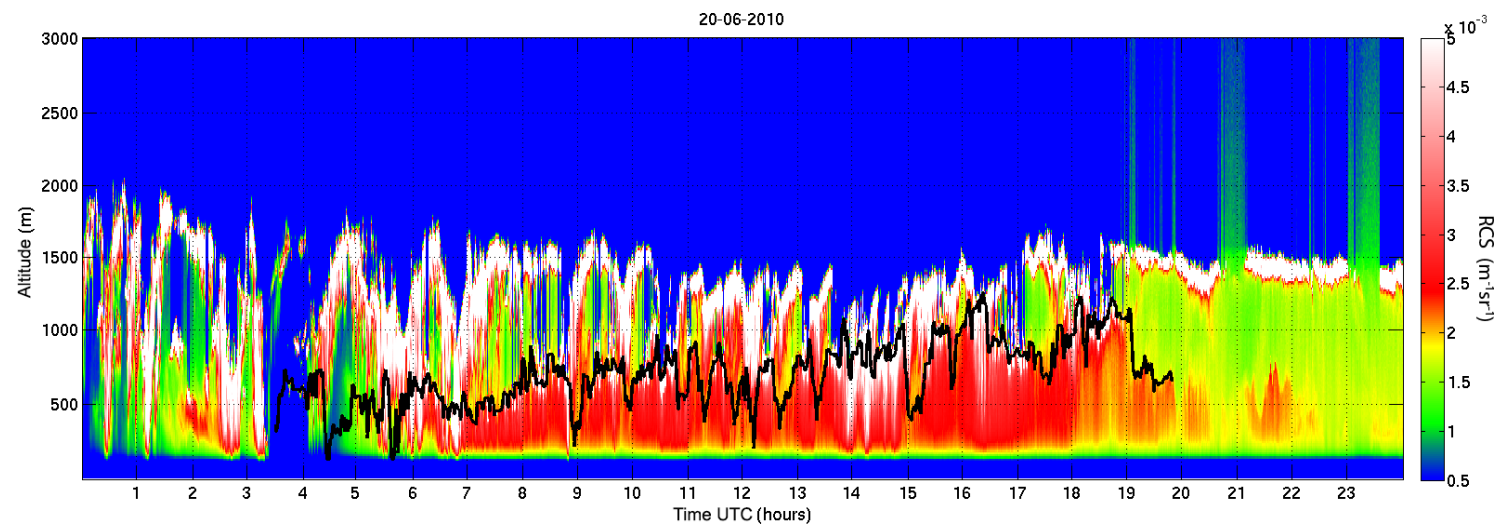

Figure 8. Lidar RCS together with pathfinder MLH estimate on 20 June 2010. A day with a completely overcast sky and precipitation falling from the cloud but (almost) completely evaporating before reaching the surface.

pears and with it the ML decreases in altitude. When the precipitation stops, air is no longer brought downward and the ML increases again in altitude. Under these conditions, the gradient on top of this layer is picked up well by the pathfinder algorithm. Similar events can be observed between 11:45 and 12:00 or 12:30 and 13:00 UTC.

\subsection{Comparison to other methods - 12-day period}

For a comparison of the lidar methods, pathfinder and STRAT2D, which were applied to the same data, were compared to radiosonde and wind profiler MLH retrievals. We used the observations from a 12-day period in May 2008, obtained during the IMPACT campaign at Cabauw. Radiosonde observations were taken around 05:00, 10:00 and 16:00 UTC each day. The Richardson bulk method was used to estimate $\mathrm{MLH}_{R}$. The wind profiler operated continuously during IMPACT, but $\mathrm{MLH}_{W}$ estimates could only be given for the above-mentioned 12-day period. The ALS450 also operated continuously and the pathfinder and STRAT2D algorithms were applied to derive MLH estimates, $\mathrm{MLH}_{L, P}$ and
$\mathrm{MLH}_{L, S}$ respectively. An overview of lidar measurements and the different MLH time series are shown in Fig. 10.

\subsubsection{Wind profiler}

The MLH estimates from the wind profiler and pathfinder algorithm are in excellent agreement, with $90 \%$ of the pathfinder MLH estimates falling within a range of $250 \mathrm{~m}$ of the wind profiler values. Pathfinder mean bias compared to wind profiler MLH estimates is as small as $-9.8 \mathrm{~m}$. Largest deviations are found at midday. However, due to the difference in approach and time resolution, it is not clear which MLH estimate is more correct. Next to that, pathfinder could not track the MLH on some of the mornings because the ML was too shallow and stayed under the detection range for a large part of the morning.

\subsubsection{STRAT2D}

STRAT2D and pathfinder differ in a number of ways, including the way in which the layers are detected; pathfinder uses a simple gradient method, whereas STRAT2D is based 

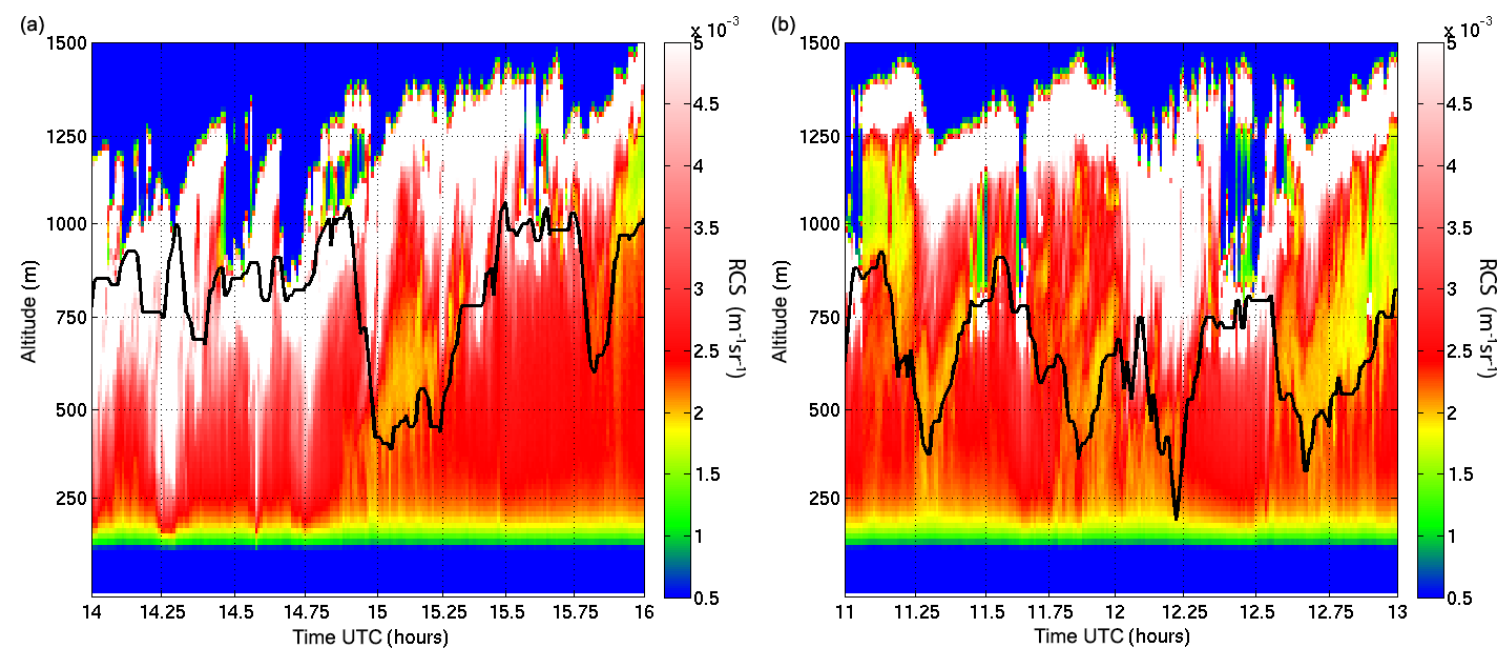

Figure 9. Highlights of the MLH estimate found by the pathfinder algorithm on 20 June 2010. See text in Sect. 4.2. Shown are (a) precipitation events reaching the lidar overlap region and (b) precipitation evaporating well above this region.

on a wavelet transform (Menut et al., 1999; Morille et al., 2007; Haeffelin et al., 2012). However, the main difference between the methods is in the ability to track the MLH over time, for which pathfinder introduces the use of graphs.

For large parts of the 12-day period, the estimates by STRAT2D and pathfinder agree well and also with the results of the wind profiler. However, for STRAT2D large differences occur between subsequent time steps when irregularities are found within the ML. This leads to unrealistic, erratic estimates of $\mathrm{MLH}_{L, S}$ evolution during the day under some conditions. This behaviour is reflected in the mean bias of $-244 \mathrm{~m}$ of STRAT2D compared to wind profiler MLH estimates: $70 \%$ of the STRAT2D estimates fall within a $250 \mathrm{~m}$ vertical range of wind profiler MLH estimates. Additionally, STRAT2D does not give an estimate due to the criterion that the ratio between backscatter above and below the MLH candidate cannot exceed a prescribed value. During the IMPACT campaign, the ML air was relatively clean, yielding too low backscatter values in the ML compared to the free atmosphere in order to fulfill the STRAT2D criterion.

\subsubsection{Radiosonde}

Agreement between radiosonde and pathfinder strongly depends on the time of day. A good correlation $\left(R^{2}=0.88\right)$ is found for the 16:00 UTC soundings excluding 14 May, with a mean bias of only $+18 \mathrm{~m}$ of pathfinder compared to radiosonde estimates and a RMSE of $156 \mathrm{~m}$. By contrast, there is little agreement at 05:00 and 10:00 UTC. At 05:00 UTC, the ML is often too low to be detected by lidar and cannot be tracked by pathfinder. Comparing the 10:00 UTC soundings with the lidar observations, it becomes clear that the Richardson bulk method often incorrectly designates the residual layer as MLH (e.g. 3, 10 and 12 May). This is reflected in a mean bias of $-938 \mathrm{~m}$ of pathfinder MLH compared to radiosonde estimates. An investigation of these 10:00 UTC soundings shows that a theoretical adiabatically rising air parcel indeed has positive buoyancy almost up to the radiosonde estimate. The Richardson bulk method estimates MLH slightly higher because wind shear is taken into account. For 3 May, a well mixed-layer extending up to an altitude of $900 \mathrm{~m}$ can be distinguished from the radiosonde temperature profile (not shown), coinciding with the pathfinder MLH estimate. The Richardson bulk method, however, incorrectly estimates the MLH at about $1600 \mathrm{~m}$. The reason may be that the Richardson bulk method does not take into account entrainment of environmental air into the rising thermal. By mixing in air with a lower (potential) temperature, the parcel loses its positive buoyancy at a lower altitude than the theoretical non-mixing rising parcel. Ignoring entrainment might be appropriate for deep convection, where the major part of the ascent takes place in non-turbulent air, but because of the turbulent nature of the ML, entrainment takes place at a much higher rate and cannot be neglected.

\subsection{Full-year analysis of midday MLH}

To quantify the performance of pathfinder for a wide range of atmospheric conditions, it has to be applied to longer time series and validated against different methods, preferably based on multiple instruments. Continuous, collocated measurements needed for this comparison are scarce though, so the 12:00 UTC radiosonde observations from De Bilt were considered. However, a check of the manually derived MLH estimates for Cabauw against $\mathrm{MLH}_{R}$ showed that the radiosonde measurements appeared to not be representative of the MLH at Cabauw $\left(R^{2}=0.64\right.$ with a RMSE of $\left.200 \mathrm{~m}\right)$. Also, the limitations of the Richardson bulk method as described in Sect. 4.3.3 may be of influence here. Therefore, manual estimates $\mathrm{MLH}_{M}$ at 12:00 UTC for all available days in 2010 

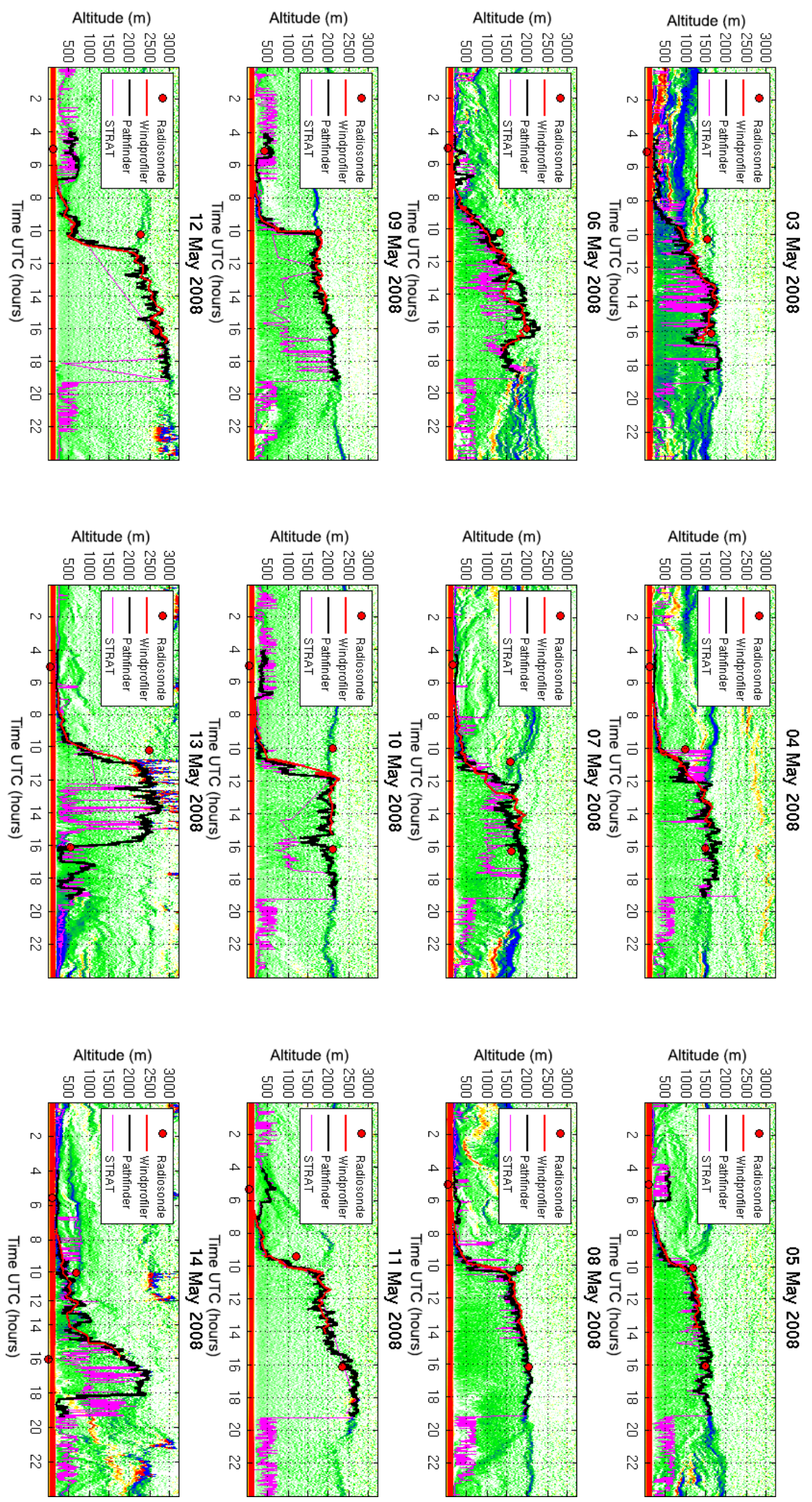

Figure 10. Overview of MLH retrievals from pathfinder $\mathrm{MLH}_{L, P}, \mathrm{STRAT}_{2} \mathrm{D} \mathrm{MLH}_{L, S}$, Wind profiler $\mathrm{MLH}_{W}$ and $\mathrm{Radiosonde} \mathrm{MLH} \mathrm{H}_{R}$ for the 12 days of IMPACT data from 2008, shown against the background of the ALS450 RCS gradient field. 
were used. Note that the pathfinder and visual/manual analysis have been performed on the exact same underlying instrument data. This ensures that only the algorithm is checked, without introducing new independent data.

$\mathrm{MLH}_{M}$ is compared to a $10 \mathrm{~min}$ average of $\mathrm{MLH}_{L, P}$ around noon. The results are shown in Fig. 11a. The correlation with the manual estimates is as high as $R^{2}=0.90$, mean bias of $-50 \mathrm{~m}$ and a RMSE of $83 \mathrm{~m}$. If the pathfinder quality criterion is used, which identifies estimates where the ratio between backscatter below and above the candidate $\mathrm{MLH}_{L, P}$ (i.e. $\left.\operatorname{RCS}\left(z>\operatorname{MLH}_{L, P}\right) / \mathrm{RCS}\left(z<\mathrm{MLH}_{L, P}\right)\right)$ is larger than 0.9, a number of days are excluded, and 197 days remain where confidence is highest. This data set yields a correlation between $\mathrm{MLH}_{M}$ and $\mathrm{MLH}_{L, P}$ of $R^{2}=0.95$, with a mean bias of $-30 \mathrm{~m}$ and RMSE of $61 \mathrm{~m}$. The STRAT2D results were also compared to the manual estimates. STRAT2D only gives an estimate if the same quality criterion is used, which leaves 234 days with a correlation of $R^{2}=0.74$, mean bias of $+43 \mathrm{~m}$ and a RMSE of $127 \mathrm{~m}$. The scatter that is larger than the pathfinder is apparent in Fig. 11b. Most of the mismatches are caused by an overestimation of the MLH by STRAT2D. In these cases STRAT2D designates the top of the residual layer as MLH. The colour coding in Fig. 11 indicates the month and reveals the seasonality. As expected, lowest MLH estimates are found in winter and highest estimates in spring and summer.

\subsection{Parameter sensitivity}

Pathfinder limits the search for MLH to altitudes near previously found MLH estimates, which gives it its improved tracking behaviour. However, the method may be sensitive to initialisation parameters. Two parameters in particular that may have an effect on the retrieved $\mathrm{MLH}_{L, P}$ time series are the start time on the day of the first time step and the time window size, i.e. the number of time steps treated simultaneously. When two possible paths have comparable total cost, including or excluding time steps in a time window might tip the outcome to either one of those competing paths. This would change the MLH estimate of that time window and possibly the outcome of subsequent time windows.

\subsubsection{Variation of the initial start time}

A change in the position of the first time step will not change the size of the search range, but shifts it along the time axis together with all other time windows of that day. As a consequence, the first MLH candidate found may be different from the one found in the default run and this may influence the subsequent MLH estimates since they are bound to the previous MLH estimates. The default time window is $15 \mathrm{~min}$, i.e. 30 time steps for the ALS450. In the test, the time window was shifted forward and backward by 1 to 30 time steps, leading to a total of one base plus 60 sensitivity runs for each day considered. The analysis was applied to the observations of May 2010. Overall, very high agreement between base and sensitivity runs is found. There is an exact agreement at least $93.1 \%$ for all individual time steps of the complete month when comparing the results of one sensitivity run with the base case. Accompanying mean bias is as low as $4.15 \mathrm{~m}$ and maximum monthly mean RMSE of $17 \mathrm{~m}$. As expected, agreement deteriorates when start and endpoints of the time windows go further away from their original position in the base run. Lowest agreement is found for a forward shift of 17 time steps.

\subsubsection{Extent of the search time window}

Within a time window, the vertical search range increases with $75 \mathrm{~m}$ both upward and downward each time step with our current settings. If the window size is increased, the search range consequently expands and more measurements are included in the search.

Next to the default time window of 30 time steps, calculations are made for window sizes between 10 and 70 time steps with increments of 10 time steps. Again, the ALS450 observations from May 2010 were used. Again, the algorithm showed stable behaviour and the exact same solution is found between 95.3 and $96.6 \%$ of the individual time steps in the month when using time windows between 20 and 70 time steps. Corresponding mean bias ranges between -7.0 and $+0.5 \mathrm{~m}$ and RMSE between 12.5 and $15.3 \mathrm{~m}$. The performance for a window size of 10 time steps was slightly worse with an exact match of $89.6 \%$, mean bias of $-21.7 \mathrm{~m}$ and RMSE of $34 \mathrm{~m}$. For this smallest time window, the maximum allowed change of the MLH of $1 \mathrm{~ms}^{-1}$ between starting and endpoint of the time window caused the algorithm to be unable to correctly track differences between individual rising thermals. As a result, many short-term differences occur between these runs and the default calculations.

All sensitivity runs with a larger time window showed the lowest correlations for a single day all on 27 May. Apart from some exceptions, this was due to the period between 14:00 and 16:00 UTC when a residual layer with clouds was in close proximity to the MLH. For window sizes larger than 30 time steps, the solution jumped from the MLH to a residual layer and followed this layer between 14:00 and 16:00 UTC. Runs with a smaller window size tracked MLH during the whole period. Pathfinder has to include several high cost time steps to allow for a jump to another layer. For relatively small time windows, there are not enough time steps left to compensate for this extra cost and the jump is rejected as a solution. In case of larger time windows, this might not be the case and the solution is allowed to jump to another layer more easily. This divergence continues as long as the tracked feature (e.g. residual or cloud layer) exists or the guiding restrictions pick up gradients forcing the calculations back to the correct solution.

Therefore, as long as the time window is large enough to capture the rising thermals, the pathfinder produces similar 
(a)

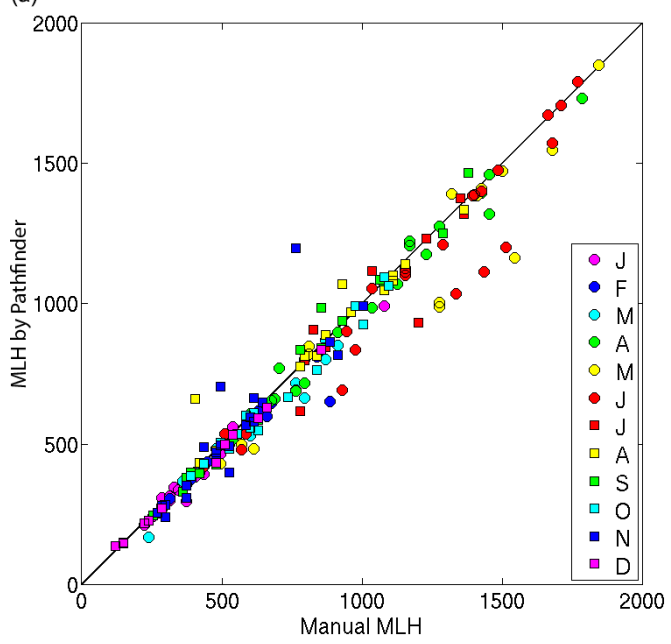

(b)

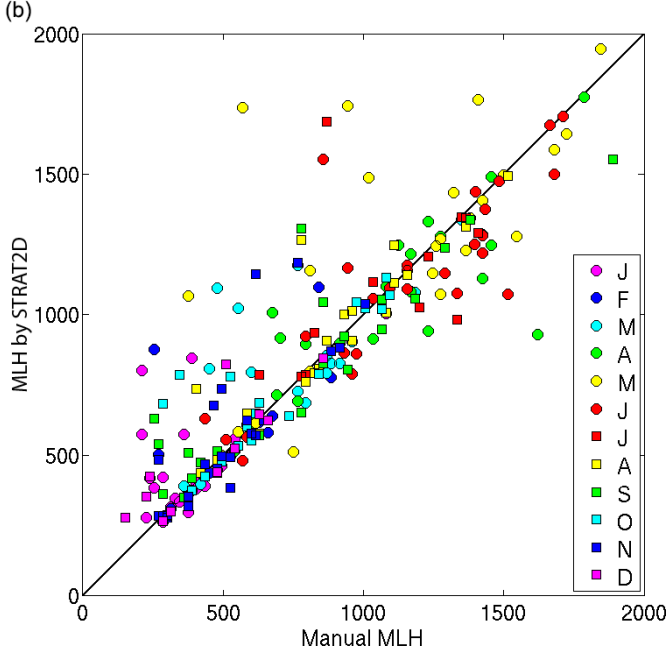

Figure 11. Scatter plots of (a) pathfinder $\mathrm{MLH}_{L, P}$ against $\mathrm{MLH}_{M}\left(R^{2}=0.96\right)$ and (b) STRAT2D MLH $\mathrm{ML}_{L}$ against $\mathrm{MLH}_{M}\left(R^{2}=0.74\right)$ for the full data set of 2010. Colour coding indicates the month of the year.

MLH estimates during a day irrespective of the time window settings. Although solutions can diverge for short periods, the guiding restrictions force the calculations back to the correct solution.

\subsection{Discussion}

The clear-sky cases show that the guiding restrictions successfully exclude large parts of the additional gradients of the residual layer. This prevents the need to apply strong smoothing filters or averaging and allows the shortest path algorithm to determine the evolution of the MLH on the native resolution of the underlying data. In case the guiding restrictions do not exclude all additional gradients, a jump to another layer of high gradients is most often prevented by the shortest path algorithm itself. For a transition, several measurement points with low gradients have to be included in the path, increasing the total cost of the jump. A transition cannot always be prevented if the additional gradients are strong enough to compensate for the extra cost. Here, further study would be needed on how to tune the algorithm settings. Also, atmospheric conditions that are different from the Dutch conditions may require different settings.

Clouds can be a good indicator of MLH height, but the high backscatter on the cloud droplets cause a negative gradient typically an order of magnitude larger than gradients associated with differences in aerosol concentration. Because of these strong gradients, the solution found by the algorithm is drawn to cloud tops. In case of a cloud layer above the ML, guidance is needed to restrict the algorithm to the MLH, for instance, by a more rigorous cloud screening prior to the pathfinder analysis.

During rain $\mathrm{MLH}_{L, P}$ derivation is difficult, because of the attenuation of the lidar signal. If precipitation did not reach the surface, it could be seen that the downdraught of a rain shower lowered MLH. Pathfinder is able to correctly follow this decrease in MLH as long as the precipitation evaporates above the overlap region $z_{f}$, otherwise there is no negative gradient to assign $\mathrm{MLH}_{L, P}$ to. The top of the precipitating cloud will be marked as $\mathrm{MLH}_{L, P}$ instead, although it is not the actual MLH.

Because of the high temporal resolution of the lidar observations, changes in MLH by individual thermals are dominant. The typical spatial scale of the thermals is of the order of several hundreds of metres up to a kilometre. To compare results to other instruments with a similar time resolution, their collocation should be better than this typical scale.

Since the MLH at night in the Netherlands is often below the minimum overlap region of the ALS450 used in this study, no attempts were made to derive the nocturnal boundary layer. Nocturnal boundary layers could be tracked if the lidar profiles would start at appropriately low heights.

Whereas pathfinder was developed specifically for MLH retrieval from stand-alone single wavelength backscatter lidar data, the method may be generalised to be applied to data from other profiling instruments for MLH retrieval. Moreover, pathfinder may be embedded in a chain of multiple algorithms, such as cloud screening. One such example has recently been described by Poltera et al. (2017).

\section{Conclusions}

A common feature in the existing methods used to derive MLH from backscatter lidar measurements based on gradient detection in aerosol loading is that MLH is derived for each time step individually, which allows for large jumps in the MLH between subsequent time steps. These jumps are not consistent with the inherent gradual evolution of the layer, which usually makes it easy for a somewhat trained 
individual to visually recognise the MLH development in lidar RCS plots. To accommodate for this, a new method was proposed called "pathfinder". This method evaluates multiple time steps within a configurable time window simultaneously. Graph theory is applied together with Dijkstra's shortest path algorithm (Dijkstra, 1959) to imitate the continuous character of the MLH.

The pathfinder algorithm stores a full day of lidar measurements arranged in a time-altitude matrix and subsequently divides the matrix into time windows of $15 \mathrm{~min}$. These 15 min blocks are translated into graphs in which each individual data point represents a vertex. To estimate MLH exactly one altitude has to be selected in each time step. For the selection a certain cost is assigned to each vertex, which is inversely proportional to the gradient at the point in the graph. This way, the path with the lowest total cost will contain the maximum sum of strong gradients and will be a good estimate for the MLH. To mimic the gradual evolution of the MLH, the distance between subsequent points is restricted. The threshold used for this is a maximum growth rate of $2.5 \mathrm{~ms}^{-1}$. Between the first and last time steps of a time window, this threshold is lowered to $1 \mathrm{~ms}^{-1}$. To guide the shortest path algorithm, a set of rules is used. Features like clouds, strong negative and positive gradients exclude parts of the observations from the search range for MLH.

Pathfinder was applied to data from a Leosphere ALS450 deployed at the Cabauw Experimental Site (CESAR) in the Netherlands. The results were checked against MLH estimates obtained from independent observations, such as those from a wind profiler and radiosondes. Excellent agreement was found between MLH estimates of the pathfinder method and from the wind profiler during a 12-day period (IMPACT campaign, May 2008). The comparison with collocated radiosonde data was more problematic, we believe, due to limitations in the Richardson bulk method. Pathfinder results were also checked against manual/visual MLH retrieval applied to the same data, as well as the results from a different algorithm, STRAT2D, applied to the same data.

In in this study, pathfinder gives less scatter than STRAT2D in the comparison of a full-year analysis with manual MLH retrievals. This is due to the jumps between layers present in the STRAT2D estimates.

The pathfinder method can be used operationally on standalone single wavelength backscatter lidar data, provided the signal-to-noise ratio is sufficient to detect aerosol layers up to a few kilometres above ground. The typical computation time is less than $5 \mathrm{~min}$ for observations from a full day, based on the Leosphere ALS450 data set. An application of pathfinder to other lidar instruments, such as the Lufft CHM15k which now being deployed in the Dutch operational observation network, is currently under investigation.

Data availability. The lidar data used in this study are publicly available from the CESAR database http://www.cesar-database.nl.
Competing interests. The authors declare that they have no conflict of interest.

Acknowledgements. The authors thank the STRAT development team for making the STRAT2D code available, for their support and fruitful discussions. The authors also thank the reviewers for their positive comments and feedback, which made it possible to improve and clarify the paper.

Edited by: L. Bianco

Reviewed by: W. Angevine and one anonymous referee

\section{References}

Angevine, W. M., White, A. B., and Avery, S. K.: Boundarylayer depth and entrainment zone characterization with a boundary-layer profiler, Bound.-Lay. Meteorol., 68, 375-385, doi:10.1007/BF00706797, 1994.

Apituley, A., Russchenberg, H., van der Marel, H., Boers, R., ten Brink, H., de Leeuw, G., Uijlenhoet, R., Arbresser-Rastburg, B., and Röckmann, T.: Overview Of Research And Networking With Ground Based Remote Sensing For Atmospheric Profiling At The Cabauw Experimental Site For Atmospheric Research (CESAR) - The Netherlands, in: Proceedings IGARSS 2008, Boston, Massachusetts, III, 903-906, 2008.

Baars, H., Ansmann, A., Engelmann, R., and Althausen, D.: Continuous monitoring of the boundary-layer top with lidar, Atmos. Chem. Phys., 8, 7281-7296, doi:10.5194/acp-8-7281-2008, 2008.

Baltink, H. K.: CESAR-database, available at: http://www. cesar-database.nl (last access: 25 May 2017), 2016.

Beyrich, F.: Mixing-height estimation in the convective boundary layer using sodar data, Bound.-Lay. Meteorol., 74, 1-18, doi:10.1007/BF00715708, 1995.

de Haij, M., Wauben, W., and Baltink, H. K.: Continuous mixing layer height determination using the LD-40 ceilometer: a feasibility study, Scientific report 2007-01, KNMI, De Bilt, 102 pp., 2007.

Dijkstra, E. W.: A note on two problems in connexion with graphs, Numer. Math., 1, 269-271, doi:10.1007/BF01386390, 1959.

Donovan, D. P. and Apituley, A.: Practical depolarization-ratiobased inversion procedure: lidar measurements of the Eyjafjallajökull ash cloud over the Netherlands, Appl. Opt., 52, 2394 2415, doi:10.1364/AO.52.002394, 2013.

Eresmaa, N., Karppinen, A., Joffre, S. M., Räsänen, J., and Talvitie, H.: Mixing height determination by ceilometer, Atmos. Chem. Phys., 6, 1485-1493, doi:10.5194/acp-6-1485-2006, 2006.

EUMETNET: E-PROFILE Website, available at: http://www. eumetnet.eu/e-profile (last access: 25 May 2017), 2016.

Flamant, C., Pelon, J., Flamant, P. H., and Durand, P.: Lidar determination of the entrainment zone thickness at the top of the unstable marine atmospheric boundary layer, Bound.-Lay. Meteorol., 83, 247-284, doi:10.1023/A:1000258318944, 1997.

Haeffelin, M., Angelini, F., Morille, Y., Martucci, G., Frey, S., Gobbi, G. P., Lolli, S., O’Dowd, C. D., Sauvage, L., XuerefRémy, I., Wastine, B., and Feist, D. G.: Evaluation of MixingHeight Retrievals from Automatic Profiling Lidars and Ceilome- 
ters in View of Future Integrated Networks in Europe, Bound.Lay. Meteorol., 143, 49-75, doi:10.1007/s10546-011-9643-z, 2012.

Harvey, N. J., Hogan, R. J., and Dacre, H. F.: A method to diagnose boundary-layer type using Doppler lidar, Q. J. Roy. Meteorol. Soc., 139, 1681-1693, doi:10.1002/qj.2068, 2013.

Hayden, K., Anlauf, K., Hoff, R., Strapp, J., Bottenheim, J., Wiebe, H., Froude, F., Martin, J., Steyn, D., and McKendry, I.: The vertical chemical and meteorological structure of the boundary layer in the Lower Fraser Valley during Pacific '93, Atmos. Environ., 31, 2089-2105, doi:10.1016/S1352-2310(96)00300-7, 1997.

Hinkley, E. D.: Laser monitoring of the atmosphere, Springer Verlag, Berlin, 1976.

Holzworth, G. C.: Estimates of mean maximum mixing depths in the contiguous United States, Mon. Weather Rev, 92, 235-242, 1964.

Hooper, W. P. and Eloranta, E. W.: Lidar Measurements of Wind in the Planetary Boundary Layer: The Method, Accuracy and Results from Joint Measurements with Radiosonde and Kytoon, J. Climate Appl. Meteorol., 25, 990-1001, doi:10.1175/15200450(1986)025<0990:LMOWIT>2.0.CO;2, 1986.

Illingworth, A.: TOPROF website, available at: http://www.toprof. imaa.cnr.it (last access: 25 May 2017), 2016.

Menut, L., Flamant, C., Pelon, J., and Flamant, P. H.: Urban boundary-layer height determination from lidar measurements over the Paris area, Appl. Opt., 38, 945-954, doi:10.1364/AO.38.000945, 1999.

Morille, Y., Haeffelin, M., Drobinski, P., and Pelon, J.: STRAT: An Automated Algorithm to Retrieve the Vertical Structure of the Atmosphere from Single-Channel Lidar Data, J. Atmos. Ocean. Technol., 24, 761-775, doi:10.1175/JTECH2008.1, 2007.

Ottersten, H.: Atmospheric Structure and Radar Backscattering in Clear Air, Radio Sci., 4, 1179-1193, doi:10.1029/RS004i012p01179, 1969.

Pal, S., Haeffelin, M., and Batchvarova, E.: Exploring a geophysical process-based attribution technique for the determination of the atmospheric boundary layer depth using aerosol lidar and nearsurface meteorological measurements, J. Geophys. Res.-Atmos., 118, 9277-9295, doi:10.1002/jgrd.50710, 2013.
Poltera, Y., Martucci, G., Collaud Coen, M., Hervo, M., Emmenegger, L., Henne, S., Brunner, D., and Haefele, A.: PathfinderTURB: an automatic boundary layer algorithm. Development, validation and application to study the impact on in-situ measurements at the Jungfraujoch, Atmos. Chem. Phys. Discuss., doi:10.5194/acp-2016-962, in review, 2017.

Roelofs, G.-J., ten Brink, H., Kiendler-Scharr, A., de Leeuw, G., Mensah, A., Minikin, A., and Otjes, R.: Evaluation of simulated aerosol properties with the aerosol-climate model ECHAM5HAM using observations from the IMPACT field campaign, Atmos. Chem. Phys., 10, 7709-7722, doi:10.5194/acp-10-77092010, 2010.

Seibert, P., Beyrich, F., Gryning, S.-E., Joffre, S., Rasmussen, A., and Tercier, P.: Review and intercomparison of operational methods for the determination of the mixing height, Atmos. Environ. 34, 1001- 1027, doi:10.1016/S1352-2310(99)00349-0, 2000.

Stull, R.: An Introduction to Boundary Layer Meteorology, Springer, 1988.

Thomas, W.: DWD Ceilometer Map, available at: http://dwd.de/ ceilomap (last access: 25 May 2017), 2016.

Van Pul, W. A. J., Holtslag, A. A. M., and Swart, D. P. J.: A comparison of ABL heights inferred routinely from lidar and radiosondes at noontime, Bound.-Lay. Meteorol., 68, 173-191, doi:10.1007/BF00712670, 1994.

Vogelezang, D. H. P. and Holtslag, A. A. M.: Evaluation and model impacts of alternative boundary-layer height formulations, Bound.-Lay. Meteorol., 81, 245-269, doi:10.1007/BF02430331, 1996.

Wiegner, M., Emeis, S., Freudenthaler, V., Heese, B., Junkermann, W., Münkel, C., Schäfer, K., Seefeldner, M., and Vogt, S.: Mixing layer height over Munich, Germany: Variability and comparisons of different methodologies, J. Geophys. Res.-Atmos., 111, doi:10.1029/2005JD006593, 2006.

Zilitinkevich, S. and Baklanov, A.: Calculation Of The Height Of The Stable Boundary Layer In Practical Applications, Bound.Lay. Meteorol., 105, 389-409, doi:10.1023/A:1020376832738, 2002. 